\title{
Trastornos de personalidad y juego patológico en adolescentes y jóvenes con dependencia de las máquinas tragamonedas
}

El objetivo de este estudio fue explorar la relación entre los patrones de personalidad desadaptativa y el juego patológico en un grupo de jugadores con dependencia de las máquinas tragamonedas. La muestra fue de 120 participantes de ambos sexos, divididos en dos grupos: 60 jugadores patológicos (edades entre 18 a 24 años) que asistieron al Programa de Cesación de Juego Patológico del Instituto de Psicología Integral del Perú (IPIP), y un grupo de 60 no jugadores de 18 a 24 años, estudiantes universitarios de Psicología de una universidad privada de Lima. Los datos fueron obtenidos mediante el Inventario Clínico Multiaxial de Millon (MCMI-II), el Cuestionario Breve de Diagnóstico del Juego Patológico (CBDJP) y la Entrevista Estructurada de la Historia de Juego. Los resultados mostraron que el $81,7 \%$ de los jugadores patológicos con adicción a máquinas tragamonedas presentaban al menos un trastorno de personalidad, a diferencia del $40 \%$ de la muestra no clínica. El trastorno de mayor prevalencia es el autoderrotista, seguido del trastorno pasivo-agresivo, trastorno narcisista y límite. Los resultados sugieren que existen diferencias marcadas entre los patrones de personalidad desadaptativa de la teoría de Millon de jugadores y no jugadores. 


\section{Personality disorders and pathological gambling in adolescents and young people with addiction to slot machines}

The object of this study was to explore the relationship between patterns of maladaptive personality and pathological gambling in a group of gamblers addicted to slot machines. The sample consisted of 120 male and female subjects divided into two groups: a group of 60 pathological gamblers aged 18 to 24 who attended the Pathological Gambling Recovery Program at the Instituto de Psicología Integral del Perú (IPIP), and a group of 60 non-gamblers aged 18 to 24 who were psychology students of a private university in Lima. Data was obtained using the Millon Clinical Multiaxial Inventory II (MCMI-II), the Pathological Gambling Brief Questionnaire (PGBQ), and the Structured Clinical Interview for Pathological Gambling (SCI-PG). The results showed that $81,7 \%$ of the pathological gamblers addicted to slot machines had at least one personality disorder, in comparison with the other group which yielded $40 \%$. The most prevalent personality disorder was the self-defeating disorder, followed by the passive-aggressive, narcissistic and borderline disorders. Moreover, there are significant differences in maladaptive personality patterns between gamblers and non-gamblers according to Millon's theory.

personality disorders / pathological gambling / slot machines 


\section{INTRODUCCIÓN}

El juego por placer, ocio o beneficios económicos tiene miles de años de antigüedad y está presente en casi todas las culturas alrededor del mundo. La búsqueda incesante de excitación y de maneras de enfrentar el aburrimiento son algunos de los factores que han predispuesto al inicio del juego a lo largo de la historia de la humanidad.

Existe una variedad de juegos que consiguen generar adicción, como las máquinas tragamonedas, los bingos, las carreras de caballos, los casinos y el póquer, entre otros. A este tipo de adicción a los juegos de azar por dinero se le conoce usualmente como juego patológico o ludopatía. Este es un problema de salud - reconocido por la American Psychiatric Association (APA) y por la Organización Mundial de la Salud (OMS) - que trae consecuencias negativas al jugador y a las personas de su entorno, pues se trata de un trastorno altamente incapacitante que afecta de forma progresiva y crónica diversas áreas de la vida de la persona que lo padece (American Psychiatric Association, APA, 2013). Estudios realizados en diversos países demuestran que el juego patológico es un problema global, e indican que entre un $70 \%$ y $90 \%$ de la población adolescente y adulta han jugado alguna vez en su vida (Abbott, Williams y Volberg, 2004; Gupta y Derevensky, 1998).

En el Perú, el juego patológico apareció como un problema social con mayor fuerza desde el año 1999, cuando se promulgó la ley que permitía la apertura de este tipo de negocios de entretenimiento, que impulsó la propagación y legalización de casinos y salas de juego, con su respectiva difusión en medios publicitarios (Ley 27153). Sin embargo, todavía es un campo poco explorado por los profesionales de la salud mental, situación que se agudiza por la desinformación hacia este tema de la población en general, que aún lo entiende como un vicio y no como una adicción. Durante mucho tiempo, la noción de adicción ha sido considerada semejante a la adicción a las drogas. Pero, si se sabe que una de las características principales de las adicciones es la falta de control y la dependencia, estas no se deben únicamente al uso de sustancias químicas, como tabaco, alcohol, marihuana, clorhidrato de cocaína, etcétera. Indistintamente, existen hábitos de conducta aparentemente inofensivos que, en determinada coyuntura, interfieren gravemente en la vida de las personas que los sufren (Echeburúa y Corral, 1994).

Aun cuando en nuestro país no se han realizado estudios epidemiológicos específicos que observen el impacto de los juegos de azar en la población en general, la prevalencia de este trastorno ha sido indagada en estudios epidemiológicos de consumo de drogas en la población general (Cedro, 2010, 2012; Devida, 2006, 2010). La IV Encuesta Nacional de Consumo de Drogas en la Población General de Perú (Devida, 2010) encontró que tres de cada diez personas (38\%) han jugado alguna vez en su vida algún juego de azar, y el 9,4\% de los jugadores es un posible jugador patológico o ludópata. Asimismo, se evidenció que existen nueve veces más usuarios recientes y actuales de juegos de azar que consumidores de sustancias ilegales, siendo el uso de drogas ilegales 2,5 veces más alto entre las personas que han jugado. Resultados similares presenta el 
Estudio epidemiológico de consumo de drogas en población urbana peruana (Cedro, 2010), en el cual se observó que el 23,6 \% alguna vez en su vida ha ido a los juegos de azar; la mayoría de ellos son mujeres (28 \%) $\mathrm{y}$, en gran parte, se ubican en un rango de edad de 19 a 24 años (30,4 \%); los juegos de azar más utilizados son las máquinas tragamonedas (12,3\%), las máquinas chinas/ bolita (3,5 \%) y los bingos (3,1\%).

En lo que se refiere a la casuística de juego patológico, las instituciones especializadas en el campo de las adicciones señalan que corresponde a problemas de adicción a los juegos de azar: máquinas tragamonedas, casinos, carreras de caballos, bingo, ruleta, etcétera. En los centros de tratamiento de las adicciones se ha demostrado un crecimiento de la demanda de ayuda por este problema. Así, el Servicio Lugar de Escucha del Centro de Información y Educación para la Prevención del Abuso de Drogas (Cedro) ha detectado en los últimos cinco años un aumento en las peticiones de ayuda por problemas de adicciones comportamentales, como la adicción a los juegos de azar. De los 1500 casos atendidos en el año 2009, alrededor del $15 \%$ son consultantes por problemas asociados al juego patológico (Rojas, 2013). Otro informe de Cedro (2012) reportó que de 245 estudiantes universitarios atendidos en el Servicio Lugar de Escucha el 11,7 \% se debía a problemas de juego patológico. Estos datos refuerzan la evidencia de que el juego patológico es una adicción que tiene que ser tomada en cuenta al momento de desarrollar programas de prevención y tratamiento en el campo de las adicciones para evitar que se convierta en un problema de salud pública (González, 1989).
Desde hace mucho tiempo, se ha desarrollado el interés por el estudio de la comorbilidad en las adicciones (Crockford y el-Guebaly, 1998; Tenorio y Marcos, 2000). El juego patológico no ha sido la excepción. En él destaca la importancia de evaluar la coexistencia con trastornos de la personalidad. Si se sabe que la tasa de trastornos de personalidad en jugadores patológicos es muy elevada, es ineludible que se ejecute un esfuerzo de investigación para el desarrollo de programas específicos de evaluación e intervención para este tipo de población. Así, un jugador patológico puro no puede ser tratado de la misma manera que un jugador con comorbilidad asociada a un trastorno de personalidad (FernándezMontalvo y Echeburúa, 2006). Además, los jugadores patológicos tienden a presentar una serie de problemas en distintas áreas de su vida: psicopatológicos, familiares, de pareja, laborales/académicos, sociales, consumo de sustancias, legales, económicos, entre otros. Para la mayor parte de las personas, el juego es una actividad normal, pero para los jugadores patológicos se convierte en una conducta que no son capaces de controlar (OMS, 1992).

Al igual que ocurre con cualquier adicción, la causalidad del juego patológico es multifactorial. Los patrones de personalidad son algunos de los factores causales y mantenedores de la adicción al juego (Black y Moyer, 1998; Blaszczynski y Steel, 1998; Fernández-Montalvo y Echeburúa, 2006; Ortiz-Tallo, Cardenal, Ferragut y Cerezo, 2011; Specker, Carlson, Edmonson, Johnson y Marcotte, 1996). Por otra parte, el intentar establecer la relación entre la personalidad y la adicción a las 
máquinas tragamonedas se fundamenta en que estas máquinas recreativas son las que tienen mayor prevalencia de vida y generan mayor número de personas con problemas de juego patológico en nuestro país y a nivel mundial (Cedro, 2010; Devida, 2006, 2010).

La falta de opciones de tratamiento especializadas en esta área y la casi inexistente investigación en el ámbito local sobre este tema hacen más difícil el abordaje terapéutico, debido a que no hay una base teórica que aporte un perfil del juego patológico en el Perú. A lo antes mencionado se suma la comorbilidad existente (demostrada en otros contextos) entre el juego patológico, el uso de drogas, los trastornos psiquiátricos, los desórdenes de personalidad, la búsqueda de nuevas sensaciones y las distorsiones cognitivas relacionadas con los juegos de azar, con énfasis en máquinas tragamonedas (Bland, Newman, Orn y Stebelsky, 1993; Feigelman, Wallisch y Lesieur, 1998).

Asimismo, diversos estudios han encontrado los efectos negativos que tiene el juego patológico en distintas esferas de la vida del jugador. En el ámbito psicológico, provoca tristeza, ansiedad, depresión e irritabilidad. En lo familiar, desatención y falta de comunicación. En la esfera laboral/ académica, causa bajo rendimiento, desmotivación, faltas injustificadas, abandono del trabajo o estudios, y despidos. En lo social, genera desatención de las amistades, disminución de las actividades de ocio, pérdida de relaciones significativas y consumo de sustancias. Esto conduce al uso y abuso de drogas legales e ilegales: tabaco, alcohol, cocaína, anfetaminas, etcétera. También puede traer problemas con la justicia, estafas, robos y otras actividades delictivas, así como problemas económicos por las deudas relacionadas con el juego (Echeburúa y Corral, 1994).

La presente investigación busca aportar al desarrollo de un modelo explicativo de la conducta de juego patológico con base en los rasgos de personalidad como factores predisponentes y de mantenimiento del juego patológico (Bland et al., 1993; Ramírez, McCormick, Russo y Taber, 1983; Roy et al., 1988; Linden, Pope y Jonas, 1986). Los resultados obtenidos en este estudio - aunque aún preliminares y provisionales - podrán constituirse en un marco de referencia para la elaboración y diseño de estrategias terapéuticas y preventivas del tratamiento del juego patológico. En cuanto a la justificación social, las personas que se encuentren en riesgo de desarrollar un problema de juego patológico y las personas con problemas de juego patológico se verán beneficiadas, ya que los resultados contribuirán a la elaboración de una propuesta de tratamiento para la cesación del juego patológico, fundada en la terapia cognitiva conductual, con énfasis en los rasgos de personalidad, la búsqueda de nuevas sensaciones y las distorsiones cognitivas.

\section{DEFINICIÓN Y CRITERIOS DIAGNÓSTICOS} DEL JUEGO PATOLÓGICO

No fue hasta 1975 que se empezó a investigar la ludopatía como enfermedad y, en 1979, Moran la definió como juego patológico. Su reconocimiento oficial se produjo en 1980, cuando la American Psychiatric Association (APA) la incluye en una de las categorías del Manual diagnóstico y estadístico de los trastornos mentales (DSM) de ese año. 
Cuando se menciona la palabra adicción, es común pensar en la "adicción a las drogas". No obstante, se ha reconocido que el factor principal de todos los trastornos adictivos es la falta de control; en otras palabras, es la falta de autocontrol de la persona afectada sobre determinada conducta. Esta, en un inicio, es placentera, pero con el tiempo necesita ejercer dicha conducta para minimizar los síntomas displacenteros que generan el no realizarla; esto se conoce como síndrome de abstinencia (Cía, 2013).

La determinación de que se padece un problema de juego patológico se basa en una serie de criterios psiquiátricos diagnósticos. Este problema está descrito en los trastornos del control de impulsos no clasificados en otros apartados, junto con problemas como la cleptomanía, el trastorno explosivo intermitente, la piromanía y la tricotilomanía (APA, 1980). En el DSM-IV-TR (APA, 2000), estuvo categorizado como un trastorno del control de los impulsos no clasificado en otros apartados, junto con la cleptomanía, la piromanía, etcétera. Sin embargo, con la aparición del DSM-V (APA, 2013), se realizan cambios en las nomenclaturas y se incluye una nueva: trastornos no relacionados a sustancias, con la cual se designa a las citadas adicciones conductuales. En este tipo de trastornos se encuentra como única patología aprobada el juego patológico o ludopatía, con un nuevo rótulo: trastorno por juego de apuestas (Cía, 2013). Como señalan Echeburúa y Báez (1990), según este manual diagnóstico, los criterios para el abuso de sustancias y para el juego patológico son básicamente los mismos, si se sustituye el juego por la sustancia adictiva, con un énfasis especial en la pérdida de control. En el DSM-IV (APA, 1994), se añade un aspecto en los criterios diagnósticos para este problema, que a nuestro entender es de gran importancia: se comienza a dar relevancia tanto a las consecuencias del juego como a los factores desencadenantes del mismo, sugiriéndose la necesidad de tratamientos tanto sintomáticos como no sintomáticos de este problema.

A pesar de existen criterios específicos para diversos trastornos, como la dependencia de sustancias psicoactivas, el juego patológico, etcétera, todos parten de los criterios de dependencia de sustancias psicoactivas, dado que en las adicciones, sean con o sin sustancia, se producen los fenómenos de pérdida de control, tolerancia, síndrome de abstinencia, entre otros. Concretamente, el DSM-IV, el DSM-IV-TR y el DSM-V (APA, 1994, 2000, 2013) utilizan los siguientes criterios para diagnosticar el juego patológico.

\section{Patrones y síndromes clínicos de la personalidad según el MCMI-II}

El diagnóstico de un trastorno de personalidad se basa en los criterios diagnósticos, es decir, un conjunto de síntomas que deben encontrarse en una persona durante un largo tiempo. Cada trastorno de personalidad tiene un conjunto de criterios que describen rasgos característicos o conductas representativas de cada persona que lo padece.

Millon y Everly (1994) definen la personalidad como la representación de rasgos cognitivos, afectivos y conductuales que persisten y se manifiestan por largos períodos de tiempo, los cuales surgen de 
disposiciones biológicas y del aprendizaje de la persona. Este concepto ha ido variando de tal manera que ahora la personalidad se determina como un patrón complicado de particularidades psicológicas profundamente arraigadas, que se expresan de manera automática en la actividad psicológica de la persona.

\section{Juego patológico en jóvenes $y$ adolescentes}

El juego patológico en poblaciones de jóvenes y adolescentes ha demostrado ser un problema de relevancia clínica. En distintos países, se han realizado estudios epidemiológicos que indican que la prevalencia en jóvenes y adolescentes se encuentra entre el 1,7 \%, según Ladouceur e Ivfireault (1988), y el $8 \%$ hallado por Lesieur et al. (1991); también se obtuvieron prevalencias de juego patológico en estudiantes de instrucción secundaria o universitaria que van entre el $4 \%$ y el $8 \%$. Es evidente que los resultados son dos o tres veces mayores que la prevalencia obtenida en la población de adultos (Zitlow, 1992), donde se encontró un 9,6 \% de jugadores adolescentes. Algunos estudios en otros países, como en Estados Unidos, estiman que entre el $3 \%$ y el $10 \%$ de los jugadores patológicos son jóvenes y adolescentes (Westphal, Rush, Stevens y Johnson, 2000). En Canadá, se encontró un 4,7 \% en esta población (Gupta y Deverensky, 1998), y en Inglaterra algunos investigadores calculan entre el 5,6\% (Fisher, 1999) y el $6 \%$ (Wood y Griffiths, 1998).

Ciertos estudios metaanalíticos, como el realizado por Stucki y Rihs-Middel (2007), se limitan a analizar la prevalencia de juego patológico en poblaciones adultas, prescindiendo de poblaciones de jóvenes y adolescentes por las altas tasas de juego patológico que estas presentan (Stinchfield, Hanson y Olson, 2006), las cuales pueden ser incluso más elevadas que entre los adultos (Shaffer, Hall y Bilt, 1999). Este hecho exige hacer un análisis más minucioso en esta población desde el enfoque de la salud pública, porque los juegos de azar han dejado de ser considerados como únicos de la población adulta. Algunos estudiosos del juego patológico, como Sarabia, Estévez y Herrero (2014), han manifestado que los juegos de azar pasan por cambios vertiginosos por la variedad de oferta $y$ accesibilidad; las nuevas tecnologías, como las computadoras y los teléfonos móviles, han abierto el acceso desde cualquier lugar geográfico a distintos artilugios relacionados con los juegos de azar, siendo los preferidos las máquinas tragamonedas.

\section{ANTECEDENTES DE LA INVESTIGACIÓN}

Los estudios realizados demuestran una alta tasa de trastornos de personalidad (TP) en jugadores patológicos. Lesieur y Blume (1990), en una investigación con 141 jugadores patológicos, encontraron que el $71 \%$ de ellos presentaba al menos un trastorno de personalidad. También Blaszczynski y Steel (1998), en una muestra de 82 participantes, determinaron que el $93 \%$ sufría un trastorno. Por su parte, el estudio de Fernández-Montalvo y Echeburúa (2006) en jugadores patológicos halló que el $32 \%$ estuvo afectado por al menos un trastorno. Black y Moyer (1998) observaron que el $87 \%$ de una muestra de 30 sujetos tenía, 
como mínimo, un trastorno de personalidad relacionado con la dependencia de los juegos de azar. Similares resultados obtuvieron Odlaug, Schreiber y Grant (2013): entre el $23 \%$ y el $92 \%$ de los jugadores patológicos padecen por lo menos un trastorno de personalidad, y el $18 \%$ de ellos manifiesta tener entre dos o más trastornos de personalidad.

Algunos autores han demostrado que el trastorno de personalidad antisocial tiene una prevalencia del 16,5\% (Blaszczynski y Steel, 1998; Ibáñez y Saiz, 2001; Pietrzak y Petry, 2005). Otros estudios han hallado cifras mayores al $35 \%$ de personas con un diagnóstico antisocial (CunninghamWilliams, Cottler, Compton y Spitznagel, 1998). Los mismos investigadores, en un estudio posterior, refieren que el $22 \%$ de jugadores patológicos con trastorno antisocial tienen problemas con el consumo de drogas (Cunningham-Williams et al., 2000). Muchos estudios han comprobado lo antes mencionado, destacando que la existencia de un trastorno de personalidad y de juego patológico genera mayores probabilidades de desarrollar adicción al alcohol o el uso de drogas (French, Maclean y Ettner, 2008; Melville, Casey y Kavanagh, 2007).

En general, no existe un acuerdo acerca de los trastornos de personalidad comórbidos con el juego patológico. Así, algunas investigaciones resaltan el trastorno esquizotípico de la personalidad (Lesieur y Blume, 1990), mientras que otros estudios señalan el trastorno de evitación (Specker et al., 1996), el límite (Blaszczynski y Steel, 1998; Fernández-Montalvo y Echeburúa, 2006; Odlaug et al., 2013), el obsesivo-compulsivo
(Black y Moyer, 1998; Odlaug et al., 2013) y el narcisista (Odlaug et al., 2013).

Para el análisis de la personalidad de los jugadores patológicos, el instrumento más utilizado es el Inventario Clínico Multiaxial de Millon (Fernández-Montalvo y Echeburúa, 2006; Henderson, 2004; Pedrero, 2009). El MCMI-II es un instrumento multiaxial derivado de un modelo integrado de la psicopatología y la personalidad, y, a su vez, está relacionado con el modelo multiaxial del DSM (Cardenal, Sánchez-López y Ortiz-Tallo, 2007).

Fernández-Montalvo y Echeburúa (2006) llevaron a cabo un estudio de los trastornos de personalidad asociados al juego patológico en una muestra conformada por 50 jugadores, que completaron el MCMI-II antes del tratamiento, y por 50 sujetos de la población normal con las mismas características demográficas. El $40 \%$ de la muestra clínica (frente al $14 \%$ de la muestra normativa) presentaba, al menos, un trastorno de personalidad. El trastorno de mayor prevalencia era el trastorno narcisista (32\%), seguido del trastorno antisocial y del trastorno pasivo-agresivo (16\% cada uno de ellos).

Ortiz-Tallo, Cancino y Cobos (2011) exploraron la presencia de patrones de personalidad y su relación con los síndromes clínicos en una muestra de 212 hombres jugadores patológicos pertenecientes a un centro de rehabilitación. Emplearon el Inventario Clínico Multiaxial de Millon (MCMI-II). Los resultados muestran que los patrones de personalidad clínicos más comunes fueron dependientes (29,7 \%), obsesivocompulsivo (29,3\%), narcisista $(23,6 \%)$ y antisocial (23,6 \%). Los síndromes clínicos 
más usuales son el abuso de alcohol (24,5\%) y el abuso de drogas (16\%).

Otro estudio, realizado por Ortiz-Tallo, Cardenal, Ferragut y Cerezo (2011), encontró relaciones significativas entre el juego patológico y el consumo de alcohol y drogas. El 25,5 \% de la muestra de jugadores patológicos tenía problemas con el alcohol y un $16 \%$ con el abuso de drogas. Otros investigadores también hallaron relaciones significativas entre la dependencia de las bebidas alcohólicas, el consumo de drogas y el juego patológico (Fernández-Montalvo y Echeburúa, 2006; Odlaug et al., 2013). Asimismo, los trastornos por abuso y dependencia de drogas legales e ilegales se encuentran fuertemente asociados al juego patológico (Grant y Kim, 2003; Welte, Barnes, Tidwell, Hoffman y Wieczorek, 2004, 2015) con tasas que fluctúan entre el $21 \%$ y el $65 \%$, tanto en población clínica como general. También se ha descubierto comorbilidad del juego patológico con otros trastornos. Los trastornos de estado de ánimo alcanzan elevadas tasas, como la depresión mayor, la distimia, la manía y la hipotimia, que oscilan entre el $33 \%$ y el $76 \%$, mientras que los trastornos de ansiedad varían entre el $9 \%$ y el $40 \%$ (Adamson, Todd y Sellman, 2006; Grant y Kim, 2003; Ibáñez y Saiz, 2001).

En el Perú, Paredes y Sandoval (2011) exploraron y describieron el perfil clínico de personalidad mediante el Inventario Clínico Multiaxial de Millon (MCMI-II) en una población de jugadores patológicos con respecto a los juegos de azar, conformada por internos en el Instituto Nacional de Salud Mental "Honorio Delgado-Hideyo
Noguchi”. El $79 \%$ de los participantes padecía al menos un trastorno de personalidad. Además, las escalas que se presentaron con mayor frecuencia y que obtuvieron puntajes elevados significativos fueron las siguientes: agresivo-sádico (52,6\%), narcisista (42,1\%), pasivo-agresivo (42,1\%), evitativo (36,8 \%) y antisocial (36,8\%). Asimismo, determinaron un promedio de 3,4 trastornos de personalidad por cada participante. Concluyeron que un gran porcentaje de pacientes con diagnóstico de juego patológico tenían comorbilidad con los trastornos de personalidad.

Los estudios mencionados anteriormente destacan el interés de valorar la posibilidad de la patología dual en grupos de jugadores patológicos dependientes de diversos juegos de azar asociados a los trastornos de personalidad, pese a que se han realizado pocos estudios que examinan la correspondencia con otros síndromes clínicos. Del mismo modo, resalta el interés por indagar la posible relación entre los perfiles de personalidad comunes a los jugadores patológicos, en los que pueden coexistir diversos trastornos de personalidad y síndromes clínicos.

\section{Objetivo}

Esta investigación tiene como objetivo explorar y comparar los rasgos desadaptativos de personalidad más relevantes en una muestra de jugadores patológicos que han buscado ayuda terapéutica, comparada con un grupo de control no clínico (estudiantes universitarios). La finalidad es reconocer los rasgos de personalidad presentes en los jugadores dependientes de las máquinas 
tragamonedas. Se espera que los resultados sean un aporte para el diseño e implementación de estrategias preventivas selectivas y procedimientos terapéuticos eficaces en función de las variables estudiadas.

\section{Método}

\section{Participantes}

El muestreo aplicado fue no probabilístico e intencional. La muestra total de este estudio estuvo conformada por 120 participantes (60 jugadores patológicos y 60 no jugadores). El grupo de jugadores patológicos estaba compuesto por 60 participantes de ambos sexos (hombres: 48,3 \% y mujeres: 51,7 \%), con edades comprendidas entre los 18 y los 24 años (edad promedio: 21,6; desviación estándar: 2,1$)$. La mayoría es soltera o soltero $(80,5$ $\%)$, tiene secundaria completa $(56,10 \%)$, su juego problema son las máquinas tragamonedas $(100 \%)$, llevan más de cuatro años de tiempo de juego $(75,1 \%)$ y con una frecuencia interdiaria $(89,4 \%)$. Los participantes acudieron en busca de tratamiento al Programa de Cesación de Juego Patológico (PCJP) del Instituto de Psicología Integral del Perú (IPIP) entre enero del 2013 a febrero del 2017.

Los criterios de inclusión fueron los siguientes: (a) ser mayores de 18 años; (b) llevar más de un año como tiempo de juego; (b) cumplir con los criterios diagnósticos de juego patológico según el DSM-V, determinados mediante la Entrevista Estructurada de la Historia de Juego; (c) alcanzar una puntuación igual o superior a 10 en el Cuestionario Breve de Diagnóstico de Juego Patológico (CBDJP); y (d) tener como juego problema las máquinas tragamonedas. $\mathrm{La}$ inclusión de los tres últimos criterios se deben al objetivo de contar con jugadores que cumplan con los criterios diagnósticos de juego patológico y homogeneizar la muestra en cuanto al tipo de juego problema.

El grupo de control estaba conformado por 60 personas no jugadoras de juegos de azar, y fue denominado población no clinica. Estaba constituido por estudiantes universitarios de la carrera de Psicología de una universidad particular de Lima Metropolitana y se encontraban cursando entre el primery el quinto año (hombres: $75, \%$ y mujeres: $25, \%$ ). La mayoría son solteros o solteras $(53,7 \%)$, con nivel educativo universitario $(100 \%)$ y edades comprendidas entre los 18 a 24 años (edad promedio: 21,3; desviación estándar: 1,9). Los criterios de inclusión fueron los siguientes: (a) mayores de 18 años; (b) no cumplir con los criterios diagnósticos de juego patológico según el DSM-V; (c) alcanzar una puntuación igual o inferior a 4 en el CBDJP; (d) no haber jugado (ni siquiera con la compra de un boleto de lotería) en el último año. Todos los participantes firmaron el consentimiento informado para formar parte de este estudio.

\section{Instrumentos}

Inventario Clínico Multiaxial de Millon (MCMI-II)

Construido por Millon (1987), es un inventario autoadministrable que consta de 175 afirmaciones breves y autodescriptivas, con alternativas de respuesta de tipo dicotómico (falso y verdadero). Fue elaborado desde el modelo estructural politético. Asimismo, 
explora dimensionalmente 13 escalas de patrones desadaptativos de personalidad. El MCMI-II está conformado por 26 escalas diagnósticas comprendidas en los patrones clínicos de personalidad (esquizoide, evitativo, dependiente, histriónico, narcisista, antisocial, agresivo-sádico, compulsivo, pasivo-agresivo y autoderrotista), patología severa de la personalidad (esquizotípico, borderline y paranoide), síndromes clínicos (ansiedad, somatoformo, bipolar, distimia, dependencia de alcohol y dependencia de drogas), síndromes severos (desorden del pensamiento, depresión mayor y desorden delusional) y escalas de fiabilidad y validez (validez, sinceridad, deseabilidad social y autodescalificación). El contenido de este inventario procede de la teoría biopsicosocial de Theodore Millon, según la cual los síndromes clínicos son extensiones de los estilos de personalidad.

En el Perú, Garibay (2002) realizó un análisis exploratorio en un grupo de 260 estudiantes de Psicología de una universidad nacional para determinar la confiabilidad y la validez de este instrumento. En cuanto a la confiabilidad, por el método de consistencia interna, los valores se situaron entre 0,42 y 0,91 ; el menor valor fue el de la escala compulsiva. Respecto a la confiabilidad, por mitades, los valores obtenidos se hallaron entre .42 y .89 , siendo el menor el de la escala dependiente. Además, se brindaron baremos asignados en puntajes T para la población estudiantil, a diferencia de las puntuaciones TB que asigna el autor del inventario.

Con la finalidad de establecer y analizar la confiabilidad del MCMI-II en el presente estudio, se procedió a analizar los resultados obtenidos con los datos de los participantes utilizando el alfa de Cronbach. Este alcanzó un valor de .93 , lo que permite concluir que el instrumento es confiable y, por lo tanto, se puede aplicar en la investigación, ya que evidencia una alta consistencia interna.

\section{Cuestionario Breve de Diagnóstico del} Juego Patológico (CBDJP)

La primera versión de este cuestionario estaba compuesta por 50 preguntas y fue desarrollada por Salinas y Roa (2001). Debido a las características del problema que evaluaba, era aconsejable elaborar un cuestionario de screening, con un número menor de ítems que el Cuestionario de Diagnóstico del Juego Patológico FAJER en el que está basado. Para cada uno de los diez criterios del DSM-IV, se seleccionaron los dos ítems con correlación ítem-total corregido más elevada y cuyo contenido fuese suficientemente diferente. Así, quedaron 20 ítems con la misma estructura de respuesta y sistema de puntuación - "Nunca", "A veces" y "Muchas veces"- que el del anterior cuestionario, por lo que la puntuación total varía entre 0 y 40. La puntuación final permite establecer "No jugador" (0 a 4), "Jugador con riesgo" (5 a 9) y "Jugador dependiente" (10 a más).

La consistencia interna, valorada mediante el coeficiente alfa de Cronbach, alcanza el valor 93 para la muestra conjunta de ambos grupos. En la muestra de jugadores, su valor es .83 y, en la población general, es .76. La validez concurrente con el cuestionario original es elevada $(r=.986)$. La puntuación discrimina claramente ambos grupos; la puntuación media de la muestra de población general es 1,19 
(s. d. =2,76), mientras que en la muestra de jugadores patológicos es 15,95 (s. d. =6,65). El punto de corte óptimo es cinco; con él se consigue una sensibilidad del 98,4 \% y una especificidad del 93,5 \% (FernándezMontalvo et al., 1995; Salinas, 2004).

Entrevista Estructurada de la Historia de Juego

La Entrevista Estructurada de la Historia de Juego (Echeburúa y Báez, 1994) consta de 10 ítems que recolectan datos sobre los comportamientos de juego de los padres, el inicio y el agravamiento de la conducta de juego en el paciente y otros aspectos de la vida cotidiana que son útiles en la evaluación y control del problema específico de cada persona.

\section{Procedimiento de recolección de datos}

La recolección de datos para la muestra de jugadores patológicos fue realizada como parte del proceso de ingreso al Programa de Cesación de Juego Patológico (PCJP) del Instituto de Psicología Integral del Perú (IPIP). En la primera sesión, se recolectó la información personal y la relacionada con el juego, que fue brindada por el consultante y corroborada por la persona acompañante. En esta etapa se aplicó la Entrevista Estructurada de la Historia de Juego, el Cuestionario Breve de Juego Patológico (CBJP) y el Inventario Clínico Multiaxial de Millon (MCMI-II), instrumentos que forman parte del proceso de evaluación psicológica. En cambio, la evaluación del grupo no clínico o de control se llevó a cabo en las aulas de clase seleccionadas, durante una sola sesión, en días y horarios previamente pactados con los docentes, evitando los momentos antes de algún examen o la última hora de clase. Se entregó la Entrevista Estructurada de la Historia de Juego, el Cuestionario Breve de Diagnóstico de Juego Patológico (CBDJP) y el Inventario Clínico Multiaxial de Millon (MCMI-II) dentro de un sobre A4 a cada estudiante. Antes de iniciar la recolección de los datos, se brindó información breve acerca del objeto de estudio y se invitó a participar de manera voluntaria y anónima a los estudiantes. En promedio, las evaluaciones en ambos grupos duraron entre 40 a 60 minutos.

\section{Estrategia de análisis}

Los datos recolectados y codificados fueron sometidos a tratamiento estadístico en el programa SPSS 24,0 (Statistical Package for the Social Sciences) para Windows. Previamente a los análisis de la base de datos, se ejecutó un proceso de consolidación, que comprende la integración de archivos, la consistencia de la información, la reestructuración de los datos, la tabulación básica, la presentación y difusión de los resultados, y la consistencia de rango de la información global y estructural. Los procedimientos estadísticos para el análisis de los datos fueron los siguientes: análisis de frecuencias para describir las características de la muestra y análisis bivariados ( $\mathrm{t}$ de Student).

Solo se tomaron en cuenta los protocolos de respuesta con puntajes significativos en la escala de validez, según los criterios del MCMI-II. Y, con arreglo a los criterios más conservadores, solo se ha considerado la presencia de trastornos de personalidad 
cuando la puntuación en la tasa base (TB) del MCMI-II era superior a 84. Para el análisis total de la muestra, únicamente se han contemplado los datos de los protocolos de respuesta con puntajes significativos en la escala de validez $(>1)$ y sinceridad (menos de 145 o más de 590), según los criterios del MCMI-II. Los instrumentos de evaluación completos son procesados y corregidos por personal independiente al estudio, lo cual garantiza mayor transparencia y objetividad en la entrada de datos.

\section{Resultados}

Las siguientes tablas contienen estadísticas descriptivas y comparativas de las escalas del MCMI-II. Solo se tomaron en cuenta los protocolos de respuesta con puntajes significativos en la escala de validez, según los criterios del MCMI-II, y con arreglo a los criterios más conservadores de Wetzler
(1990), únicamente se ha considerado la presencia de trastornos de personalidad cuando la puntuación en la tasa base (TB) del MCMI-II era superior a 84. Para el análisis total de la muestra, se contemplan exclusivamente los datos de los protocolos de respuesta con puntajes significativos en la escala de validez $(>1)$ y sinceridad (menos de 145 o más de 590), según los criterios del MCMI-II.

En la tabla 1, se puede observar la frecuencia y los porcentajes de patrones clínicos y patología severa de personalidad en los dos grupos estudiados, según fueron diagnosticados con el MCMI-II. Se considera la existencia de un trastorno de personalidad solo cuando la tasa base del MCMI-II es mayor a 84. El 81,7\% de los jugadores patológicos tienen por lo menos un rasgo desadaptativo de la personalidad, comparado con el grupo no clínico (40\%).

\section{Tabla 1}

Frecuencia y porcentajes de patrones clínicos y patología severa de la personalidad en el grupo de jugadores patológicos, comparados con los grupos de control, según el MCMI-II

\begin{tabular}{llc}
\hline \multicolumn{1}{c}{ Muestras } & \multicolumn{2}{c}{$\begin{array}{c}\text { Patrones clínicos y patología severa de la } \\
\text { personalidad }\end{array}$} \\
\cline { 2 - 3 } & $\mathrm{N}$ & $\%$ \\
\hline Jugadores patológicos $(\mathrm{N}=60)$ & 49 & 81,7 \\
Población no clínica $(\mathrm{N}=60)$ & 24 & 40,0 \\
\hline
\end{tabular}


En la tabla 2, se evidencia el porcentaje de patrones clínicos y patología severa de personalidad en jugadores y no jugadores. Se puede apreciar que el $18,3 \%$ de los jugadores no tiene trastornos de personalidad, al igual que el $40 \%$ de la población no clínica. En la muestra de jugadores patológicos, el $81,7 \%$ padece entre uno a más de un trastorno por persona, a diferencia del $60 \%$ de los no jugadores, que tienen entre uno y dos trastornos de personalidad.

\section{Tabla 2}

Porcentajes de patrones clínicos y patología severa de personalidad en el grupo de jugadores patológicos y población no clínica, según el MCMI-II

\begin{tabular}{lcc}
\hline Trastornos & Jugadores patológicos & Población no clínica \\
\cline { 2 - 3 } & $\%$ & $\%$ \\
\hline 0 & 18,3 & 40,0 \\
2 & 6,7 & 45,0 \\
3 & 16,7 & 15,0 \\
4 & 18,3 & 0,0 \\
5 & 13,3 & 0,0 \\
6 & 3,3 & 0,0 \\
7 & 10,0 & 0,0 \\
8 & 5,0 & 0,0 \\
9 & 1,7 & 0,0 \\
Más de 10 & 17 & 0,0 \\
\hline
\end{tabular}

Nota: Solo se ha considerado la puntuación en la tasa base (TB) del MCMI-II superior a 84.

En la tabla 3, se evidencia que el trastorno de mayor prevalencia es el trastorno autoderrotista (40\%), seguido del trastorno pasivo-agresivo (38,3\%), el trastorno narcisista $(36,7 \%)$, límite $(36,7 \%)$, evitativo $(31,7 \%)$, antisocial (30\%) y dependiente
(25 \%). En el grupo de no jugadores, las tasas más altas se dan en el trastorno compulsivo $(51,7 \%)$, dependiente $(15 \%)$, histriónico y agresivo-sádico (cada uno con $3,3 \%$ ), y narcisista (1,7\%), no evidenciándose ninguno de los demás trastornos. 
Tabla 3

Frecuencia y porcentajes de patrones clínicos y patología severa de personalidad en jugadores patológicos y no jugadores $(N=120)$

\begin{tabular}{lcccc}
\hline \multicolumn{1}{c}{ Escalas } & \multicolumn{2}{c}{ Jugadores patológicos } & \multicolumn{2}{c}{ No jugadores } \\
\cline { 2 - 5 } Patrones clínicos & $\mathrm{N}$ & $\%$ & $\mathrm{~N}$ & $\%$ \\
\hline Esquizoide & 11 & 18,3 & 0 & 0,0 \\
Evitativo & 19 & 31,7 & 0 & 0,0 \\
Dependiente & 15 & 25,0 & 9 & 15,0 \\
Histriónico & 10 & 16,7 & 2 & 3,3 \\
Narcisista & 22 & 36,7 & 1 & 1,7 \\
Antisocial & 18 & 30,0 & 0 & 0,0 \\
Agresivo-sádico & 17 & 28,3 & 2 & 3,3 \\
Compulsivo & 10 & 16,7 & 31 & 51,7 \\
Pasivo-agresivo & 23 & 38,3 & 0 & 0,0 \\
Autoderrotista & 24 & 40,0 & 0 & 0,0 \\
\hline Patología severa & $\mathrm{N}$ & $\%$ & $\mathrm{~N}$ & $\%$ \\
\hline Esquizotípico & 7 & 11,7 & 0 & 0,0 \\
Límite & 22 & 36,7 & 0 & 0,0 \\
Paranoide & 9 & 15,0 & 0 & 0,0 \\
\hline
\end{tabular}

Nota: Solo se ha considerado la puntuación en la tasa base (TB) del MCMI-II superior a 84 .

En la tabla 4, se presentan los síndromes clínicos y severos de mayor prevalencia en el grupo de jugadores patológicos: en primer lugar está la ansiedad (43,3\%), seguida de la distimia (41,7\%), la dependencia del alcohol (15\%) y la dependencia de drogas $(13,3 \%)$. En el grupo de no jugadores no se encontró ningún trastorno. 
Tabla 4

Frecuencia y porcentajes de síndromes clínicos y síndromes severos de personalidad en jugadores patológicos y no jugadores $(N=120)$

\begin{tabular}{lllll}
\hline Escalas & \multicolumn{2}{l}{ Jugadores patológicos } & \multicolumn{2}{l}{ No jugadores } \\
\cline { 2 - 5 } Síndromes clínicos & $\mathrm{N}$ & $\%$ & $\mathrm{~N}$ & $\%$ \\
\hline Ansiedad & 26 & 43,3 & 0 & 0,0 \\
Somatoforme & 5 & 8,3 & 0 & 0,0 \\
Bipolar & 5 & 8,3 & 0 & 0,0 \\
Distimia & 25 & 41,7 & 0 & 0,0 \\
Dependencia del alcohol & 9 & 15,0 & 0 & 0,0 \\
Dependencia de drogas & 8 & 13,3 & 0 & 0,0 \\
\hline Síndromes severos & & & & 0,0 \\
\hline Desorden de pensamiento & 5 & 8,3 & 0 & 0,0 \\
Depresión mayor & 6 & 10,0 & 0 & 0,0 \\
Desorden delusional & 4 & 6,7 & 0 & \\
\hline
\end{tabular}

Nota: Solo se ha considerado la puntuación en la tasa base (TB) del MCMI-II superiores a 84 .

En la tabla 5, se exponen las diferencias entre los patrones clínicos y patología severa de personalidad en jugadores patológicos y no jugadores. Se pueden observar diferencias significativas en las puntuaciones promedio de las siguientes escalas: esquizoide $(\mathrm{t}=6,19, \mathrm{p}$ [bilateral $]=.000)$, evitativo $(\mathrm{t}=14,21, \mathrm{p}$ [bilateral $]=.000)$, histriónico $(\mathrm{t}=3,68, \mathrm{p}$ [bilateral] $=.000)$, narcisista $(\mathrm{t}=6,41, \mathrm{p}$ [bilateral $]=.000)$, antisocial $(\mathrm{t}=10,68, \mathrm{p}$ [bilateral] $=.000)$, agresivosádico $(\mathrm{t}=6,13, \mathrm{p}$ [bilateral] $=.000)$, compulsivo $(\mathrm{t}=-6,41, \mathrm{p}$ [bilateral] $=.000)$, pasivo-agresivo $(\mathrm{t}=14,81, \mathrm{p}$ [bilateral] $=.000)$, autoderrotista $(\mathrm{t}=11,74, \mathrm{p}$ [bilateral] $=.000)$, esquizotípico $(\mathrm{t}=7,93, \mathrm{p}$ [bilateral] $=.000)$, límite $(\mathrm{t}=15,12, \mathrm{p}$ [bilateral] $=.000)$ y paranoide $(\mathrm{t}=4,41, \mathrm{p}$ [bilateral] $=.000)$. No se encontraron diferencias significativas en las puntuaciones promedio entre ambos grupos en la escala de dependencia $(\mathrm{t}=-1,58, \mathrm{p}$ [bilateral $]=.117)$.

Del mismo modo, en los patrones clínicos y patología severa de personalidad en el grupo de jugadores patológicos, se obtuvieron indicadores moderados en las escalas: evitativo $(75,9)$, narcisista $(79,8)$, antisocial $(77,2)$, agresivo-sádico $(80,7)$, pasivo-agresivo $(76,2)$, autoderrotista $(77,9)$ y borderline $(78,0)$. Asimismo, se evidencian en este grupo indicadores sugestivos en las escalas esquizoide $(70,6)$, dependiente $(71,3)$, histriónico $(71,0)$, compulsivo $(69,9)$, esquizotípico $(68,0)$ y paranoide $(70,5)$. En el grupo de no jugadores, se hallaron indicadores moderados en la escala de compulsivo $(84,7)$ y agresivo-sádico $(62,1)$.

En la tabla 6, se observan las diferencias entre ambos grupos (jugadores patológicos 
Tabla 5

Puntuaciones de los patrones clínicos y patología severa de personalidad en jugadores patológicos y no jugadores: $t$ de Student, media aritmética y desviación estándar $(N=120)$

\begin{tabular}{|c|c|c|c|c|c|c|c|c|}
\hline \multirow{2}{*}{ Escalas } & \multicolumn{3}{|c|}{ Jugadores patológicos } & \multicolumn{3}{|c|}{ No jugadores } & \multirow{2}{*}{$\mathrm{t}$} & \multirow{2}{*}{ d } \\
\hline & $\mathrm{N}$ & Media & DS & $\mathrm{N}$ & Media & DS & & \\
\hline
\end{tabular}

Patrones clínicos de personalidad

\begin{tabular}{lcccccccc}
\hline Esquizoide & 60 & 70,6 & 17,9 & 60 & 48,8 & 20,6 & $6,19^{* *}$ & 1,13 \\
Evitativo & 60 & 75,9 & 22,1 & 60 & 21,9 & 19,5 & $14,21^{* *}$ & 2,59 \\
Dependiente & 60 & 71,3 & 20,6 & 60 & 65,5 & 19,0 & 1,58 & 0,29 \\
Histriónico & 60 & 71,0 & 20,2 & 60 & 57,6 & 19,9 & $3,68^{* *}$ & 0,67 \\
Narcisista & 60 & 79,8 & 16,2 & 60 & 59,9 & 17,8 & $6,41^{* *}$ & 1,17 \\
Antisocial & 60 & 77,2 & 18,5 & 60 & 44,1 & 15,3 & $10,68^{* *}$ & 1,95 \\
Agresivo-sádico & 60 & 80,7 & 19,5 & 60 & 62,1 & 13,2 & $6,13^{* *}$ & 1,12 \\
Compulsivo & 60 & 69,9 & 14,2 & 60 & 84,7 & 10,8 & $-6,41^{* *}$ & 1,17 \\
Pasivo-agresivo & 60 & 76,2 & 28,2 & 60 & 15,1 & 14,9 & $14,81^{* *}$ & 2,71 \\
Autoderrotista & 60 & 77,9 & 26,8 & 60 & 29,7 & 17,0 & $11,74^{* *}$ & 2,15 \\
\hline Patología severa de personalidad & & & & & & \\
\hline Esquizotípico & 60 & 68,0 & 17,2 & 60 & 42,2 & 18,5 & $7,93^{* *}$ & 1,36 \\
Límite & 60 & 78,0 & 23,3 & 60 & 22,5 & 16,3 & $15,12^{* *}$ & 2,58 \\
Paranoide & 60 & 70,5 & 15,4 & 60 & 59,1 & 12,8 & $4,41^{* *}$ & 0,66 \\
\hline
\end{tabular}

Nota: ${ }^{* *} p<.001$

y no jugadores) con relación a los síndromes clínicos y severos de personalidad del MCMI-II. Se hallaron diferencias significativas en las puntuaciones promedio de todas las escalas: ansiedad $(\mathrm{t}=13,38$, $\mathrm{p}$ [bilateral] $=.000)$, somatoforme $(\mathrm{t}=3,76$, $\mathrm{p}$ [bilateral] $=.000)$, bipolar $(\mathrm{t}=4,75$, $\mathrm{p}$ [bilateral $]=.000)$, distimia $(\mathrm{t}=13,09$, $\mathrm{p}$ [bilateral] $=.000)$, dependencia del alcohol $(\mathrm{t}=13,50, \mathrm{p}$ [bilateral] $=.000)$, dependencia de $\operatorname{drogas}(\mathrm{t}=9,73, \mathrm{p}$ [bilateral $]=.000)$, desorden del pensamiento $(\mathrm{t}=7,11, \mathrm{p}$ [bilateral $]=.000)$, depresión mayor $(\mathrm{t}=12,20, \mathrm{p}$ [bilateral] $=.000) \mathrm{y}$ desorden delusional $(\mathrm{t}=4,10$, $\mathrm{p}$ [bilateral $]=.003)$.

Igualmente, se advierten indicadores sugestivos en los puntajes en las escalas de los síndromes clínicos y severos de personalidad en el grupo de jugadores patológicos: ansiedad $(73,2)$, somatoforme $(62,3)$, bipolar $(62,2)$, distimia $(70,4)$, dependencia del alcohol $(67,4)$, dependencia de drogas 
$(68,2)$, desorden de pensamiento $(64,1)$, depresión mayor $(62,8)$ y desorden delusional $(62,2)$. No se encontraron indicadores significativos de ningún síntoma clínico y síndrome severo de personalidad en el grupo de no jugadores.

Tabla 6

Puntuaciones de los síntomas clínicos y síndromes severos de personalidad en jugadores patológicos y no jugadores: $t$ de Student, media aritmética y desviación estándar $(N=120)$

\begin{tabular}{|c|c|c|c|c|c|c|c|c|}
\hline \multirow{2}{*}{ Escalas } & \multicolumn{3}{|c|}{$\begin{array}{l}\text { Jugadores } \\
\text { patológicos }\end{array}$} & \multicolumn{3}{|c|}{ No jugadores } & \multirow{2}{*}{$\mathrm{t}$} & \multirow{2}{*}{$d$} \\
\hline & $\mathrm{N}$ & Media & DS & $\mathrm{N}$ & Media & DS & & \\
\hline \multicolumn{9}{|l|}{ Síntomas clínicos } \\
\hline Ansiedad & 60 & 73,2 & 27,7 & 60 & 23,8 & 7,1 & $13,38^{* *}$ & 2,44 \\
\hline Somatoforme & 60 & 62,3 & 17,6 & 60 & 49,9 & 18,3 & $3,76^{* *}$ & 0,69 \\
\hline Bipolar & 60 & 62,2 & 18,2 & 60 & 47,18 & 14,4 & $4,75^{\star *}$ & 0,92 \\
\hline Distimia & 60 & 70,4 & 26,7 & 60 & 24,2 & 5,8 & $13,09^{* *}$ & 2,39 \\
\hline $\begin{array}{l}\text { Dependencia } \\
\text { del alcohol }\end{array}$ & 60 & 67,4 & 17,2 & 60 & 28,7 & 14,1 & $13,50^{* *}$ & 2,46 \\
\hline $\begin{array}{l}\text { Dependencia } \\
\text { de drogas }\end{array}$ & 60 & 68,2 & 17,6 & 60 & 39,0 & 15,2 & $9,73^{* *}$ & 1,78 \\
\hline \multicolumn{9}{|l|}{ Síndromes severos } \\
\hline $\begin{array}{l}\text { Desorden } \\
\text { de pensamiento }\end{array}$ & 60 & 64,1 & 16,9 & 60 & 37,4 & 23,6 & $7,11^{* *}$ & 1,30 \\
\hline Depresión mayor & 60 & 62,8 & 21,7 & 60 & 15,7 & 20,4 & $12,20^{* *}$ & 2,24 \\
\hline Desorden delusional & 60 & 62,2 & 12,9 & 60 & 51,9 & 14,5 & $4,10^{* *}$ & 0,75 \\
\hline
\end{tabular}

Nota: ${ }^{* *} p<.001$

\section{CONCLUSIONES}

En el presente estudio, se encontró una alta tasa $(82 \%)$ de trastornos de personalidad en los jugadores patológicos con adicción a las máquinas tragamonedas, quienes presentaban al menos un trastorno de personalidad, un poco más que el doble (40\%) de la muestra de no jugadores. Estos resultados son muy similares al $71 \%$ obtenido por Lesieur y Blume (1990), y el 79 \% del estudio realizado por Paredes y Sandoval (2011); también está cerca del $87 \%$ evidenciado en la investigación de Black y Moyer (1998). Sin embargo, la tasa de trastornos de personalidad de este estudio es 
más elevada que la determinada por otros estudios, como el $25 \%$ de Specker et al. (1996), y el 40 \% que arrojó el estudio de Fernández-Montalvo y Echeburúa (2006). No obstante, encaja en los parámetros establecidos por Odlaug et al. (2013), que indican que entre el $23 \%$ y el $92 \%$ de los jugadores patológicos manifiestan por lo menos un trastorno de personalidad.

La falta de consistencia de estos resultados puede estar relacionada con los instrumentos utilizados para la recolección de datos y la diversidad de las muestras de jugadores patológicos (tipo de juego, frecuencia de juego, intensidad de juego, etcétera). Asimismo, puede reflejar la tendencia de sobrediagnosticar trastornos de personalidad cuando se emplean instrumentos de autoinforme (FernándezMontalvo y Echeburúa, 2006).

Por otra parte, en este estudio, el promedio de trastornos de personalidad diagnosticados para cada participante de la muestra de jugadores patológicos es de 3 , a diferencia de un trastorno en la población no jugadora. Estos datos son similares a los obtenidos por otros estudios, como el 3,4 de Paredes y Sandoval (2011), y el 2,2 de Fernández-Montalvo y Echeburúa (2006) en muestras de jugadores patológicos.

De hecho, el trastorno de mayor prevalencia es el trastorno autoderrotista de la personalidad, que afecta al $40 \%$ de los jugadores de la muestra, seguido del trastorno pasivo-agresivo (38,3\%). Estos resultados son similares al 35,4\% de Blaszczynski y Steel (1998), y al 42,1 \% de Paredes y Sandoval (2011). En cuanto al trastorno narcisista, el $36,7 \%$ encontrado en este estudio se asemeja a la tasa porcentual (32\%) hallada por Fernández-Montalvo y Echeburúa (2006), y al 42,1 \% del estudio de Paredes y Sandoval (2011). El patrón clínico de personalidad límite $(36,7 \%)$ de esta investigación difiere del 15,8\% determinado por Paredes y Sandoval (2011) y del $16 \%$ de los resultados obtenidos por Fernández-Montalvo y Echeburúa (2006).

El trastorno de personalidad evitativo presenta una tasa de 31,7\%, similar al $35 \%$ encontrado por Cunningham-Williams et al. (1998); asimismo, se asemeja a la tasa de $36,8 \%$ de Paredes y Sandoval (2011), y al 36,6 \% de Blaszczynski y Steel (1998). Igualmente, se encontró que el patrón clínico de personalidad antisocial (30\%) tiene una prevalencia parecida $(36,8 \%)$ en los estudios de Paredes y Sandoval (2011), y un $29,3 \%$ en el estudio de Blaszczynski y Steel (1998). El trastorno de personalidad dependiente en el presente estudio ha obtenido un porcentaje del $25 \%$, muy parecido al 21,1 \% de Paredes y Sandoval (2011), también en una muestra de jugadores. En cuanto al patrón de personalidad compulsiva, el porcentaje es del 16,7 \%, resultado coherente con el $15,8 \%$ obtenido por Paredes y Sandoval (2011), y el $14 \%$ de Lesieur y Blume (1990).

Cabe señalar que la presencia en la muestra no clínica de los trastornos dependiente y compulsivo se asemeja a lo expuesto en otro estudio que comparó dos muestras: una de jugadores $\mathrm{y}$ otra de no jugadores (Fernández-Montalvo y Echeburúa, 2006). El análisis de los datos indica diferencias significativas en la mayoría de las escalas de los patrones 
clínicos y patología severa de personalidad del Inventario Clínico Multiaxial de Millon (MCMI-II) al comparar las muestras de jugadores patológicos y no jugadores. Los resultados mantienen relación con otras investigaciones que señalaron diferencias en los perfiles de personalidad de jugadores patológicos y no jugadores (FernándezMontalvo y Echeburúa, 2006; Henderson, 2004). Así, se comprueba la correlación existente entre los patrones clínicos y patología severa de personalidad en el grupo de jugadores y no jugadores: autoderrotista $(\mathrm{t}=11,74, \mathrm{p}$ [bilateral] $=.000)$; esquizotípico $(\mathrm{t}=7,93, \mathrm{p}$ [bilateral] $=.000)$, borderline $(\mathrm{t}=15,12, \mathrm{p}$ [bilateral] $=.000)$ y paranoide $(\mathrm{t}=4,41, \mathrm{p}$ [bilateral] $=.000)$. No se encontraron diferencias significativas en el grupo de jugadores patológicos dependientes de las de máquinas tragamonedas, pero sí se pueden observar diferencias significativas en las puntuaciones promedio de las siguientes escalas: esquizoide $(\mathrm{t}=6,19$, $\mathrm{p}$ [bilateral] $=.000)$; evitativo $(\mathrm{t}=14,21$, $\mathrm{p}$ [bilateral] $=.000)$, histriónico $(\mathrm{t}=3,68$, $\mathrm{p}$ [bilateral] $=.000)$, narcisista $(\mathrm{t}=6,41, \mathrm{p}$ [bilateral $]=.000)$, antisocial $(\mathrm{t}=10,68, \mathrm{p}$ [bilateral] $=.000)$, agresivo-sádico $(\mathrm{t}=6,13$, $\mathrm{p}$ [bilateral] $=.000)$, compulsivo $(\mathrm{t}=-6,41$, $\mathrm{p}$ [bilateral] $=.000)$, pasivo-agresivo $(\mathrm{t}=$ $14,81, \mathrm{p}$ [bilateral] $=.000$ ), y puntuaciones medias entre ambos grupos en la escala de dependencia $(\mathrm{t}=1,58, \mathrm{p}$ [bilateral] $=.117)$.

Del mismo modo, en los patrones clínicos y patología severa de personalidad en el grupo de jugadores patológicos se observan indicadores moderados en las escalas agresivo-sádico $(80,7)$, narcisista $(79,8)$, borderline $(78,0)$, autoderrotista $(77,9)$, antisocial $(77,2)$, pasivo-agresivo $(76,2)$ y evitativo $(75,9)$. En este mismo grupo, también se advierten indicadores sugestivos: dependiente $(71,3)$, histriónico (71), esquizoide $(70,6)$, paranoide $(70,5)$, compulsivo $(69,9)$ y esquizotípico (68). En el grupo de no jugadores, se encontraron indicadores sugestivos en las escalas dependiente $(65,5)$ y agresivo-sádico $(62,1)$, y solo indicadores moderados en compulsión $(84,7)$. Los resultados coinciden con los de diversos estudios que indagaban en la correlación de los trastornos de personalidad y el juego patológico (Blaszczynski y Steel, 1998; Black y Moyer, 1998; Fernández-Montalvo y Echeburúa, 2006; Henderson, 2004; Lesieur y Blume, 1990; Specker et al., 1996).

Existe evidencia clara que relaciona el juego patológico con los comportamientos psicopáticos como las conductas desviadas e ilegales (Cunningham-Williams et al., 1998; Ibáñez y Saiz, 2001; Pietrzak y Petry, 2005). Este estudio no es la excepción; al correlacionar las puntuaciones promedio del grupo de jugadores patológicos en la escala antisocial $(77,2)$ y del grupo de no jugadores $(44,1)$, se hallaron diferencias significativas entre ambas $(\mathrm{t}=10,68, \mathrm{p}$ [bilateral $]=.000)$.

Un dato interesante es el de la escala de dependencia del alcohol, en donde se observan indicadores sugestivos en el grupo de jugadores $(67,4)$, a diferencia de los no jugadores $(28,7)$, y se advierte una diferencia significativa en el análisis de ambos grupos $(\mathrm{t}=13,5, \mathrm{p}$ [bilateral] $=.000)$. Algo similar ocurre en la escala dependencia de drogas, en donde se encontraron indicadores significativos $(68,2)$ para el grupo de jugadores, a diferencia del grupo de no jugadores $(39,9)$; asimismo, se nota 
una diferencia estadísticamente significativa en la dependencia de drogas en ambos grupos $(\mathrm{t}=9,7, \mathrm{p}$ [bilateral] $=.000)$. Estos resultados podrían indicar una correlación entre el consumo de alcohol y drogas asociado al uso de juegos de azar, en este caso, máquinas tragamonedas con premio, lo que coincide con lo planteado en estudios anteriores en una población similar (French et al., 2008; Melville et al., 2007).

Estudios realizados en el extranjero $\mathrm{y}$ en población de jugadores patológicos (Adamson et al., 2006; Grant y Kim, 2003; Ibáñez y Saiz, 2001) encontraron que los trastornos de ansiedad oscilan entre el $9 \%$ y el $40 \%$. Este resultado es similar al de este estudio, que halló un 27,7 \% en los síntomas clínicos de ansiedad en la población de jugadores; sin embargo, también se notan indicadores sugestivos de trastornos de ansiedad $(73,2)$. Aun cuando en este estudio se obtuvo un $21,7 \%$ en la depresión mayor para el grupo de jugadores, lo que difiere del $33 \%$ al $76 \%$ establecido en otros estudios, los resultados mostraron indicadores sugestivos en depresión mayor $(62,8)$. Al comparar los grupos de jugadores patológicos y de no jugadores en cuanto a los síntomas clínicos y síndromes severos de personalidad, no se observaron diferencias significativas en ninguna de las escalas de personalidad.

No obstante, los resultados refieren que existen diferencias manifiestas entre los patrones de personalidad desadaptativa de la teoría de Millon entre los jugadores patológicos y los no jugadores. Estos resultados son confirmados por otros investigadores que reconocen la presencia de trastornos, sean estos previos o comórbidos con otros trastornos, los cuales significan un riesgo claro de agravamiento psicopatológico, que se evidencia en un peor pronóstico, evolución más deficiente e inconvenientes a la hora de efectuar programas terapéuticos (Gándara y Medina, 1998; Kosten, Kosten y Rounsaville, 1989; Seivewright y Daly, 1997).

\section{RECOMENDACIONES}

En definitiva, la presente investigación presenta algunas limitaciones, debido a que se utilizó una muestra pequeña y no probabilística. Futuras investigaciones deberían contrastar los perfiles de personalidad entre grupos de jugadores de otros tipos de juegos de azar (bingo, póquer, ruleta, etcétera), así como también grupo de jóvenes y adolescentes con otros grupos etarios (adultos, adultos mayores). También sería conveniente comparar patrones de juego como el tiempo y la frecuencia de juego. Asimismo, el uso de una prueba de autoinforme para la evaluación de trastornos de personalidad (MCMI-II) puede reflejar la tendencia de sobrediagnosticar trastornos de personalidad.

Este estudio busca aportar mayor evidencia en una muestra de jugadores patológicos con la finalidad de comparar los cuadros clínicos de los jugadores de otros países con una muestra peruana. Esto se realiza considerando los pocos estudios en nuestro país que exploran los perfiles de personalidad en la población de jugadores patológicos, especialmente en jóvenes y adolescentes.

Los resultados, aun cuando son exploratorios, sugieren que existen diferencias marcadas entre los patrones de personalidad desadaptativa, sean estos previos o 
comórbidos con otros trastornos, lo que implica un riesgo claro de agravamiento psicopatológico, un pronóstico reservado y complicaciones a la hora de implementar programas terapéuticos. Por lo antes mencionado, es importante tener en cuenta estas diferencias en el proceso de consejería, orientación y tratamiento del juego patológico en poblaciones especiales, como son los jóvenes y adolescentes.

\section{REFERENCIAS}

Abbott, M., Williams, M., y Volberg, R. (2004). A Prospective Study of Problem and Regular Nonproblem Gamblers Living in the Community. Substance Use \& Misuse, 39, 855-84.

Adamson, S., Todd, F., y Sellman, J. (2006). Co-Existing Psychiatric Disorders in a New Zealand Outpatient's Alcohol and Other Drug Clinical Population. Australian and New Zealand Journal of Psychiatry, 40,164-170.

American Psychiatric Association (APA). (1980). Diagnostic and Statistical Manual of Mental Disorders (3. ${ }^{a}$ ed.). Washington, D. C.: Autor.

American Psychiatric Association (APA). (1987). Diagnostic and Statistical Manual (3. ${ }^{a}$ ed. revisada). Washington, D. C.: Autor.

American Psychiatric Association (APA). (1994). Diagnostic and Statistical Manual of Mental Disorders (4. ${ }^{\mathrm{a}}$ ed.). Washington, D. C.: Autor.

American Psychiatric Association (APA). (2013). Manual diagnóstico y estadístico de los trastornos mentales (5. ${ }^{\mathrm{a}}$ ed.). Arlington, VA: American Psychiatric Publishing.

Black D., y Moyer T. (1998). Clinical Features and Psychiatric Comorbidity of Subjects with Pathological Gambling Behavior. Psychiatric Service, 49, 1434-1439.

Bland, R., Newman, S., Orn, H., y Stebelsky, G. (1993). Epidemiology of Pathological Gambling in Edmonton. Canadian Journal of Psychiatry, 38, 108-112.

Blaszczynski, A., y Steel, Z. (1998). Personality Disorders among Pathological Gamblers. Journal of Gambling Studies, 14, 51-71.

Blaszczynsky, A., Wilson, A., y McConaghy, N. (1986). Sensation Seeking and Pathological Gambling. British Journal of Addiction, 81, 113-120.

Cardenal, V., Sánchez-López, M., y Ortiz-Tallo, M. (2007). Los trastornos de personalidad según el modelo de Millon: una propuesta integradora. Clínica y Salud, 18, 305-324.

Centro de Información y Educación para la Prevención del Abuso de Drogas (Cedro). (2010). Estudio epidemiológico de consumo de drogas en población urbana peruana 2010. Lima: Autor. 
Centro de Información y Educación para la Prevención del Abuso de Drogas (Cedro). (2012). Estudio de universitarios consumidores de drogas y abuso de juego atendidos en Cedro 2011. Recuperado de http://www.slideshare.net/lescucha/cedropresentaunpri merestudiosobreconsumodedrogasyjuegosenlneaen-universitarios

Cía, A. (2013). Las adicciones no relacionadas a sustancias (DSM-5, APA, 2013): un primer paso hacia la inclusión de las adicciones conductuales en las clasificaciones categoriales vigentes. Revista de Neuropsiquiatría, 76, 210-217.

Comisión Nacional para el Desarrollo y Vida sin Drogas (Devida). (2006). III Encuesta Nacional de Consumo de Drogas en la Población General de Perú, 2006. Lima: Autor.

Comisión Nacional para el Desarrollo y Vida sin Drogas (Devida). (2010). IV Encuesta Nacional de Consumo de Drogas en la Población General de Perú, 2010. Lima: Autor.

Crockford, D., y el-Guebaly, N. (1998). Psychiatric Comorbidity in Pathological Gambling: A Critical Review. Canadian Journal of Psychiatry, 43, 43-50.

Cunningham-Williams, R., Cottler, L., Compton, W., y Spitznagel, E. (1998). Taking Chances: Problem Gamblers and Mental Health Disorders. Results from the St. Louis Epidemiologic Catchment Area Study. American Journal of Public Health, 88, 1093-1096.

Cunningham-Williams, R., Cottler, L., Compton, W., Spitznagel, E., y Ben-Abdallah, A. (2000). Problem Gambling, Comorbid Psychiatric, and Substance Use Disorders among Drug Users Recruited from Drug Treatment and Community Settings. Journal of Gambling Studies, 16, 347-376.

Echeburúa, E., y Corral, P. (1994). Adicciones psicológicas: más allá de la metáfora. Clínica y Salud, 5, 251-258.

Echeburúa, E., y Báez, C. (1994). Concepto y evaluación del juego patológico. En J. L. Graña (Ed.), Conductas adictivas. Teoría, evaluación y tratamiento (pp. 521-556). Madrid: Debate.

Feigelman, W., Wallisch, L., y Lesieur, H. (1998). Problem Gamblers, Problem Substance Users, and Dual Problem Individuals: An Epidemiological Study. American Journal of Public Health, 88, 467-470.

Fernández-Montalvo, J., y Echeburúa, E. (2006). Juego patológico y trastornos de personalidad: un estudio piloto con el MCMI-II. Psicothema, 3, 453-457.

Fernández-Montalvo, J., Echeburúa, E., y Báez, C. (1995). El Cuestionario Breve de Juego Patológico: un nuevo instrumento de screening. Análisis y Modificación de Conducta, 21, 211-223.

Fisher, S. (1999). Prevalence Study of Gambling and Problem Gambling in British Adolescents. Addiction Research, 7, 509-538. 
French, M., Maclean, J., y Ettner, S. (2008). Drinkers and Bettors: Investigating the Complementarity of Alcohol Consumption and Problem Gambling. Drug and Alcohol Dependence, 96, 155-164.

Gándara, J., y Medina, M. (1998). Comorbilidad. En M. Bernardo y M. Roca (Eds.), Trastornos de la personalidad. Evaluación y tratamiento (pp. 97-122). Barcelona: Masson.

Garibay, C. (2002). Análisis exploratorio psicométrico del Inventario Clínico Multiaxial de Millon II en estudiantes de psicología (tesis de licenciatura). Universidad Nacional Federico Villarreal.

González, A. (1989). Juego patológico: una nueva adicción. Madrid: Tibidabo.

Grant, J., y Kim, S. (2003). Comorbidity of Impulse-Control Disorders among Pathological Gamblers. Acta Psychiatrica Scandanavia, 108, 207-213.

Gupta R., y Derevensky J. (1998). Adolescent Gambling Behavior: A Prevalence Study and Examination of the Correlates Associated with Problem Gambling. Journal of Gambling Studies, 14, 319-345.

Kosten, T. A., Kosten, T. R., y Rounsaville, B. (1989). Personality Disorders in Opiate Addicts Show Prognostic Specificity. Journal of Substance Abuse Treatment, 6, 163-168.

Henderson, M. (2004). Psychological Correlates of Comorbid Gambling in Psychiatric Outpatients: A Pilot Study. Substance Use \& Misuse, 39, 1341-1352.

Ibáñez, A., y Saiz, J. (2001). La ludopatía: una “nueva” enfermedad. Barcelona: Masson.

Ladouceur, R., e Ivfireault, C. (1988). Crumbling Behaviors among High School Students in the Quebec Area. The Journal of Gambling Behavior, 4, 3-12.

Lesieur, H., y Blume, S. (1990). Characteristics of Pathological Gamblers Identified among Patients on a Psychiatric Admission Service. Hospital and Community Psychiatry, 41, 1009-1012.

Lesieur, H., Cross, J., Frank, M., Welch, M., White, C., Rubenstein, G., y Mark. M. (1991). Gambling and Pathological Gambling among University Students. Addictive Behaviors, 16, 517-527.

Linden, M., Pope, H., y Jonas, J. (1986). Pathological Gambling and Major Affective Disorder: Preliminary Findings. Journal of Clinical Psychiatry, 47, 201-203.

Ley 27153. Ley que regula la explotación de los juegos de casino y máquinas tragamonedas (9 de julio de 1999). Recuperado del sitio web del Ministerio de Comercio Exterior y Turismo: http://www.mincetur.gob.pe/turismo/DGJCMT/index.htm

Melville, K., Casey, L., y Kavanagh, D. (2007). Psychological Treatment Dropout among Pathological Gamblers. Clinical Psychology Review, 27, 944-958. 
Millon, T. (1987). MCMI-II. Millon Clinical Multiaxial Inventory-II. Mineápolis, MN: National Computer Systems.

Millon, T., Davis, R., Millon, C., Escobar, L., y Meagher, S. (2001). Trastornos de la personalidad en la vida moderna. Barcelona: Masson.

Millon, T., y Everly, G. (1994). La personalidad y sus trastornos. Barcelona: Martínez Roca.

Organización Mundial de la Salud (OMS). (1992). CIE-10. Décima revisión de la clasificación internacional de las enfermedades, trastornos mentales y del comportamiento. Descripciones clínicas y pautas para el diagnóstico. Madrid: Méditor.

Odlaug, B., Schreiber, L., y Grant, J. (2013). Personality Dimensions and Disorders in Pathological Gambling. Current Opinion in Psychiatry, 26, 107-112.

Ortiz-Tallo, M., Cancino, C., y Cobos, S. (2011). Juego patológico, patrones de personalidad y síndromes clínicos. Adicciones, 23, 189-197.

Ortiz-Tallo, M., Cardenal, V., Ferragut, M., y Cerezo, M. (2011). Personalidad y síndromes clínicos. Un estudio con el MCMI-III basado en una muestra española. Revista de Psicopatología y Psicología Clínica, 16, 49-59.

Paredes, S., y Sandoval, Y. (2011). Perfil clínico de personalidad mediante el Inventario Clínico Multiaxial (MCMI-II) en población ludópata del Instituto Nacional de Salud Mental "Honorio Delgado-Hideyo Noguchi”. Revista Científica de Ciencias de la Salud, 4, 5-13.

Pedrero, E. (2009). Dimensiones de los trastornos de personalidad en el MCMI-II en adictos a sustancias en tratamiento. Adicciones, 21, 29-37.

Pietrzak, R., y Petry, N. (2005). Antisocial Personality Disorder is Associated with Increased Severity of Gambling, Medical, Drug, and Psychiatric Problems among TreatmentSeeking Pathological Gamblers. Addiction, 100, 1183-1193.

Ramírez, L., McCormick, R., Russo, A., y Taber, J. (1983). Patterns of Substance Abuse in Pathological Gamblers Undergoing Treatment. Addictive Behavior, 8, 425-428.

Rojas, M. (2013). Epidemiología nacional del juego, uso de internet y redes sociales virtuales en el Perú y fundamentos clínicos. Lima: Cedro; CONCYTEC.

Roy, A., Ardinoff, B., Roehrich, L., Lamparski, D., Custer, R., y Lorenz, V. (1988). Pathological Gambling. A Psychobiological Study. Archives of General Psychiatry, 45, 369-373.

Salinas, J. (2004). Instrumentos de diagnóstico y screening del juego patológico. Salud y Drogas, 4, 35-59.

Salinas, J., y Roa, J. (2001). Cuestionario de Diagnóstico del Juego Patológico FAJER. Revista Internacional de Psicología Clínica y de la Salud, 1, 353-370. 
Sarabia, I., Estévez, A., y Herrero, D. (2014). Perfiles de jugadores patológicos en la adolescencia. Revista Española de Drogodependencias, 2, 46-58.

Seivewright, N., y Daly, C. (1997). Personality Disorder and Drug Use: A Review. Drug and Alcohol Review, 76, 235-250.

Specker, S., Carlson, G., Edmonson, K., Johnson, P., y Marcotte, M. (1996). Psychopathology in Pathological Gamblers Seeking Treatment. Journal of Gambling Studies, 12, 67-82.

Shaffer, H., Hall, M., y Bilt, J. (1999). Estimating the Prevalence of Disordered Gambling Behavior in the United States and Canada. A Research Synthesis. American Journal of Public Health, 89, 1369-1376.

Stinchfield, R., Hanson, W., y Olson, D. (2006). Problem and Pathological Gambling among College Students. New Directions for Student Services, 113, 63-72.

Stucki, S., y Rihs-Middel, M. (2007). Prevalence of Adult Problem and Pathological Gambling between 2000 and 2005: An Update. Journal of Gambling Studies, 23, 245-257.

Tenorio, J., y Marcos, J. (2000). Trastornos duales: tratamiento y coordinación. Papeles del Psicólogo, 77, 58-63.

Welte, J., Barnes, G., Tidwell, M., Hoffman, J., y Wieczorek, W. (2004). The Relationship of Ecological and Geographic Factors to Gambling Behavior and Pathology. Journal of Gambling Studies, 20, 405-423.

Welte, J., Barnes, G., Tidwell, M., Hoffman, J., y Wieczorek, W. (2015). Gambling and Problem Gambling in the United States: Changes between 1999 and 2013. Journal of Gambling Studies, 3, 695-715.

Wetzler, S. (1990). The Millon Clinical Multiaxial Inventory (MCMI). A Review. Journal of Personality assessment, 55, 445-464.

Westphal, J., Rush, J., Stevens, L., y Johnson, L. (2000). Gambling Behavior of Louisiana Students in Grades 6 through 12. Psychiatric Services, 51, 96-99.

Wood, R., y Griffiths, M. (1998). The Acquisition, Development, and Maintenance of Lottery and Scratchcard Gambling in Adolescence. Journal of Adolescence, 21, 265-273.

Zitlow, D. (1992). Incidence and Comparative Study of Compulsive Gambling Behaviors between Indian and Non-Indians Within and Near a Northern Plains Reservation. Bemidji, MN: Indian Health Service, Bemidji Area Office. 